\title{
DOMAIN BLOCH CONSTANTS
}

BY

C. DAVID MINDA

\begin{abstract}
The classical Bloch constant $\mathscr{B}$ is defined for holomorphic functions $f$ defined on $\mathbf{B}=\{z:|z|<1\}$ and normalized by $\left|f^{\prime}(0)\right|=1$. Let $R_{f}$ denote the Riemann surface of $f$ and $B_{f}$ the set of branch points. Then $\mathscr{B}$ can be regarded as a lower bound for the radius of the largest disk contained in $R_{f} \backslash B_{f}$. The metric on $R_{f}$ used to measure the size of disks on $R_{f}$ is obtained by lifting the euclidean metric from $\mathbf{C}$ to $R_{f}$. The surface $R_{f}$ can also be regarded as spread over $\mathbf{B}$ and the hyperbolic metric lifted to $R_{f}$. One may then ask for the radius of the largest hyperbolic disk on $R_{f} \backslash B_{f}$. A lower bound for this radius is called a domain Bloch constant. The determination of domain Bloch constants is nontrivial for nonconstant analytic functions $f: \mathbf{B} \rightarrow X$, where $X$ is a hyperbolic Riemann surface. Upper and lower bounds for domain Bloch constants are given. Also, domain Bloch constants are given an interpretation as a radius of local schlichtness.
\end{abstract}

1. Introduction. Let $f: \mathbf{B} \rightarrow X$ be an analytic function, where $\mathbf{B}=\{z:|z|<1\}$ and $X$ is a hyperbolic Riemann surface; that is, $\mathbf{B}$ is the universal covering surface of $X$. Let $f^{X}(0) \in[0,1]$ denote the "derivative" of $f$ at the origin relative to the hyperbolic metric on both $\mathbf{B}$ and $X$. For $\alpha \in(0,1]$ we demonstrate that there is a positive constant $\mathcal{T}_{1, X}(\alpha)$, not depending on $f$, such that if $f^{X}(0) \geqslant \alpha$, then $f$ is locally schlicht in some hyperbolic ball of radius $\log \left(1+\sigma_{1, X}(\alpha)\right) /\left(1-\sigma_{1, X}(\alpha)\right)$. Actually, we refine this result by introducing constants $\mathscr{T}_{m, X}(\alpha)$, where the positive integer $m$. depends on the multiplicity with which $f$ assumes values. We obtain a lower bound for $\mathscr{T}_{m, X}(\alpha)$ by making use of a differential-geometric method that was introduced by Ahlfors [1] and refined by Heins [5]. The method involves an extension of Schwarz' lemma to certain metrics. In case $X=\mathbf{B}$ we obtain lower bounds for $\mathcal{T}_{m, \mathbf{B}}(\alpha)$ by suitably generalizing the example of Ahlfors and Grunsky [3] that was used to give the conjectured sharp upper bound for the classical Bloch constant. Finally, we mention the open problem of obtaining bounds on the constants $\delta_{m, X}(\alpha)$ which have the property that if $f^{X}(0) \geqslant \alpha$, then $f$ is schlicht in some hyperbolic ball of radius $\log \left(1+\delta_{m, X}(\alpha)\right) /\left(1-\delta_{m, X}(\alpha)\right)$.

2. Preliminaries. In this section we establish some notation and terminology that will be employed throughout this paper. We also establish one lemma.

Definition. Let $X, Y$ be Riemann surfaces. For $m \in \mathbf{Z}^{+} \cup\{\infty\}$ let $\mathscr{F}_{m}(X, Y)$ be the family of all nonconstant analytic functions $f: X \rightarrow Y$ such that for any $q \in f(X)$ each root of $f=q$ is either simple or else has multiplicity at least $m+1$.

Received by the editors October 29, 1981 and, in revised form, March 12, 1982.

1980 Mathematics Subject Classification. Primary 30C80; Secondary 30C25. 
Clearly, $\mathscr{F}_{1}(X, Y)$ is just the family of all nonconstant analytic functions $f: X \rightarrow Y$ and $\mathscr{F}_{\infty}(X, Y)$ is the subfamily of locally schlicht analytic functions. Clearly, $\left\{\mathscr{F}_{m}(X, Y)\right\}_{m=1}^{\infty}$ is a decreasing sequence of families of functions and

$$
\mathscr{F}_{\infty}(X, Y)=\bigcap_{m=1}^{\infty} \mathscr{F}_{m}(X, Y) .
$$

We generally take $X=\mathbf{B}$ in this paper. The families $\mathscr{F}_{m}(X, Y)$ were also considered in [9] and [10].

Definition. For $z, w \in \mathbf{B}$ let

$$
\delta(z, w)=|(z-w) /(1-\bar{w} z)| .
$$

Also, for $z \in \mathbf{B}$ and $r \in(0,1]$ let $B(z, r)=\{w \in \mathbf{B}: \delta(z, w)<r\}$.

The function $\delta$ defines a distance on $B$ that is invariant under the group Aut(B) of conformal automorphisms of B [12, pp. 510-511]. The Poincaré hyperbolic distance $d$ on $\mathbf{B}$ is related to $\delta$ by

$$
d(z, w)=\log (1+\delta(z, w)) /(1-\delta(z, w)), \quad \delta(z, w)=\tanh (d(z, w) / 2) .
$$

We find it more convenient to work with the distance function $\delta$ in this paper. Of course, due to the relationship between $d$ and $\delta$, any result involving $\delta$ can easily be translated into one involving $d$.

Definition. Let $X$ be a Riemann surface and $f \in \mathscr{F}_{1}(\mathbf{B}, X)$. For $z \in \mathbf{B}$ let $t(z, f)$ denote the maximum value of $r \in(0,1]$ such that $f$ is locally schlicht in $B(z, r)$; set $t(z, f)=0$ in case $f$ is not locally schlicht in any neighborhood of $z$. Let $t(f)=$ $\sup \{t(z, f): z \in \mathbf{B}\}$.

It is not difficult to show that $t(z, f)$ is a continuous function; in fact, $\mid t(z, f)-$ $t(w, f) \mid \leqslant \delta(z, w)$. Also, $t(z, f)=1$ for all $z \in \mathbf{B}$ if and only if $f \in \mathscr{F}_{\infty}(\mathbf{B}, X)$.

Finally, we require certain facts about metrics on Riemann surfaces. We briefly state these facts; for details the reader should consult [9] which also contains references to other sources. Given a hyperbolic Riemann surface $X$, let $\lambda_{X}(z)|d z|$ denote the hyperbolic metric on $X$. It has constant curvature -1. In particular,

$$
\lambda_{\mathbf{B}}(z)|d z|=2|d z| /\left(1-|z|^{2}\right) \text {. }
$$

Given $f \in \mathscr{F}_{1}(X, Y)$, where $Y$ is a hyperbolic Riemann surface, let $f^{*}\left(\lambda_{Y}(z)|d z|\right)$ denote the pull-back to $X$ via $f$ of the hyperbolic metric on $Y$. This defines a metric on $X$. If $X$ and $Y$ are plane regions, then

$$
f^{*}\left(\lambda_{Y}(z)|d z|\right)=\lambda_{Y}(f(z))\left|f^{\prime}(z)\right||d z| \text {. }
$$

For $f \in \mathscr{F}_{1}(\mathbf{B}, X)$ and $a \in \mathbf{B}$ define

$$
f^{X}(a)=\left.\frac{f^{*}\left(\lambda_{X}(z)|d z|\right)}{\lambda_{\mathbf{B}}(z)|d z|}\right|_{a}
$$

This measures the local distortion of $f$ at $a$ relative to the metrics $\lambda_{X}(z)|d z|$ and $\lambda_{\mathbf{B}}(z)|d z|$. In case $X$ is a hyperbolic plane region,

$$
f^{X}(a)=\frac{1}{2} \lambda_{X}(f(a))\left|f^{\prime}(a)\right|\left(1-|a|^{2}\right) .
$$

The principle of hyperbolic metric implies that $f^{X}(a) \in[0,1]$ and $f^{X}(a)=1$ if and only if $f$ is an analytic universal covering of $\mathbf{B}$ onto $X$ [8]. 
Definition. Let $X$ be a hyperbolic Riemann surface. For $\alpha \in(0,1]$ and $m \in \mathbf{Z}^{+}$ $\cup\{\infty\}$ let

$$
\mathscr{T}_{m, X}(\alpha)=\inf \left\{t(f): f \in \mathscr{F}_{m}(\mathbf{B}, X) \text { and } f^{X}(0) \geqslant \alpha\right\} .
$$

Clearly, $\mathcal{T}_{m, X}(\alpha) \leqslant 1$ and it is not difficult to show that $\mathscr{T}_{m, X}(\alpha)$ is an increasing function of both $m$ and $\alpha$. Trivially, $\mathcal{T}_{\infty, X}(\alpha)=1$ for all $\alpha \in(0,1]$. From Theorem 2 it will follow that $\sigma_{m, X}(\alpha)$ increases to 1 as $m$ tends to $\infty$ for each fixed value of $\alpha$. The number $\mathscr{T}_{m, X}(\alpha)$ is called a domain Bloch constant; a justification for the use of this name is presented in the next section. The main purpose of this paper is to give bounds for $\mathscr{T}_{m, X}(\alpha)$. We shall obtain a lower bound for $\mathscr{T}_{m, X}(\alpha)$ by making use of a differential-geometric method that was introduced by Ahlfors [1] and refined by Heins [5]. We shall make use of the notion of an ultrahyperbolic metric; this concept is due to Ahlfors $[1,2]$. The more general concept of an $S K$ metric was introduced by Heins [5]; we shall also make use of $S K$ metrics.

LEMMA 1. Suppose that $\rho(z)|d z|$ and $\sigma(z)|d z|$ are positive $C^{2}$ metrics on a plane region $\Omega$ which have curvature at most -1 . Then the metric $\rho^{\lambda}(z) \sigma^{1-\lambda}(z)|d z|$ has curvature at most -1 for $\lambda \in(0,1)$. If $\rho^{\lambda}(z) \sigma^{1-\lambda}(z)|d z|$ has constant curvature -1 in $\Omega$, then $\rho(z)|d z|=\sigma(z)|d z|$ and these metrics also have constant curvature -1 in $\Omega$.

Proof. Set $\tau(z)|d z|=\rho^{\lambda}(z) \sigma^{1-\lambda}(z)|d z|$. The curvature of $\tau(z)|d z|$ at $z$ is

$$
\kappa(z)=-(\Delta \log \tau(z)) / \tau^{2}(z) .
$$

The curvature condition on both $\rho(z)|d z|$ and $\sigma(z)|d z|$ is equivalent to

$$
\Delta \log \rho(z) \geqslant \rho^{2}(z), \quad \Delta \log \sigma(z) \geqslant \sigma^{2}(z) .
$$

Thus,

$$
\Delta \log \tau(z)=\lambda \Delta \log \rho(z)+(1-\lambda) \Delta \log \sigma(z) \geqslant \lambda \rho^{2}(z)+(1-\lambda) \sigma^{2}(z) .
$$

Now, the inequality of the arithmetic and geometric means gives

$$
\lambda \rho^{2}(z)+(1-\lambda) \sigma^{2}(z) \geqslant \rho^{2 \lambda}(z) \sigma^{2(1-\lambda)}(z)=\tau^{2}(z),
$$

with equality if and only if $\rho(z)=\sigma(z)$. Thus

$$
\Delta \log \tau(z) \geqslant \tau^{2}(z),
$$

or $\tau(z)|d z|$ has curvature at most -1 . If $\tau(z)|d z|$ has constant curvature -1 , then we have equality in the arithmetic-geometric mean inequality and $\rho(z)|d z|$ and $\sigma(z)|d z|$ both have constant curvature -1 .

3. Relationship to usual Bloch constants. Suppose $f$ is holomorphic and nonconstant in B. Let $R_{f}$ denote the Riemann surface of $f$. Specifically, $R_{f}=\{(z, f(z)): z$ $\in \mathbf{B}\}$ is the graph of $f$. Define

$$
\begin{array}{ll}
\pi_{1}: R_{f} \rightarrow \mathbf{B}, & \pi_{2}: R_{f} \rightarrow \mathbf{C}, \\
\pi_{1}(z, f(z))=z, & \pi_{2}(z, f(z))=f(z) .
\end{array}
$$

$R_{f}$ is endowed with the unique conformal structure that makes both $\pi_{1}$ and $\pi_{2}$ into analytic functions. Note that $\pi_{1}$ is actually a conformal mapping of $R_{f}$ onto $\mathbf{B}$.

The functions $\pi_{1}$ and $\pi_{2}$ can be used to induce metrics and distance functions on $R_{f}$. Let $\lambda_{\mathbf{C}}(z)|d z|=|d z|$ denote the euclidean metric' on $\mathbf{C}$. We use this notation 
even though $\mathbf{C}$ is not a hyperbolic region. Set

$$
\rho_{1}(z)|d z|=\pi_{1}^{*}\left(\lambda_{\mathbf{B}}(z)|d z|\right), \quad \rho_{2}(z)|d z|=\pi_{2}^{*}\left(\lambda_{\mathbf{C}}(z)|d z|\right) .
$$

Then $\rho_{1}(z)|d z|$ is the hyperbolic metric on $R_{f}$. Let $B_{f}=\left\{(z, f(z)): f^{\prime}(z)=0\right\} ; B_{f}$ is the set of branch points of $R_{f}$ when viewed as spread over $C$. The metric $\rho_{i}(z)|d z|$ induces a distance function $d_{i}$ on $R_{f}$ that is compatible with the topology. For $(z, f(z)) \in R_{f}$ let $r_{i}(z, f(z))$ denote the minimum of the distance from $(z, f(z))$ to $B_{f}$ and the distance from $(z, f(z))$ to the ideal boundary of $R_{f}$ relative to the distance function $d_{i}$. Then $t(z, f)=\tanh \left(\frac{1}{2} r_{1}(z, f(z))\right)$. Also, when we view $R_{f}$ as being spread over $\mathbf{C}, r_{2}(z, f(z))$ is the radius of the largest unramified disk in $R_{f}$ with center $(z, f(z))$. Let $r_{2}(f)=\sup \left\{r_{2}(z, f(z)): z \in \mathbf{B}\right\}$. This quantity is involved in the definition of various Bloch constants [9]. The definition of these Bloch constants is parallel to the definition of the domain Bloch constants given $\$ 2$. The only difference is that the definition of the classical Bloch constants employs the pull-back of the euclidean metric on the range of the function, while the domain Bloch constants can be regarded as defined in terms of the pull-back of the hyperbolic metric on the domain of the function.

4. Lower bound for $\mathcal{T}_{m, X}(\alpha)$. We shall derive a distortion theorem which gives an upper bound for $f^{X}(z)$ in terms of $t(z, f)$ when $f \in \mathscr{F}_{m}(\mathbf{B}, X)$ and $X$ is a hyperbolic Riemann surface. This will immediately lead to a lower bound for the constant $\mathcal{T}_{m, X}(\alpha)$.

The proof of the distortion theorem requires the construction of a special metric of constant curvature -1 that is defined in a deleted neighborhood of an arbitrary point $c \in \mathbf{B}$. We briefly outline the construction of this metric; the reader is referred to [9] for more details. For $R>0$ and $m \in \mathbf{Z}^{+}$let

$$
\sigma_{m}(z)|d z|=\frac{2 R^{1 /(m+1)}|d z|}{(m+1)|z|^{m /(m+1)}\left(R^{2 /(m+1)}-|z|^{2 /(m+1)}\right)} .
$$

If $p_{m}(z)=R z^{m+1}$, then the metric $\sigma_{m}(z)|d z|$ is uniquely determined by the requirement that

$$
p_{m}^{*}\left(\sigma_{m}(z)|d z|\right)=\lambda_{\mathbf{B}}(z)|d z| \text {. }
$$

In particular, this implies that $\sigma_{m}(z)|d z|$ has constant curvature -1 on the punctured disk $\{z: 0<|z|<R\}$. The special metric that we require is obtained by shifting $\sigma_{m}(z)|d z|$ to an arbitrary point in B by means of a hyperbolic motion and then selecting $R$ appropriately. For $c \in \mathbf{B}$ let

$$
T_{c}(z)=(z-c) /(1-\bar{c} z) .
$$

Then $T_{c}$ is a conformal automorphism of $\mathbf{B}$ and

$$
T_{c}^{*}\left(\sigma_{m}(z)|d z|\right)=\sigma_{m}\left(T_{c}(z)\right) \frac{1-\left|T_{c}(z)\right|^{2}}{1-|z|^{2}}|d z|=\theta_{m}(\delta(z, c)) \lambda_{\mathbf{B}}(z)|d z|,
$$

where

$$
\theta_{m}(t)=\frac{\left(1-t^{2}\right) R^{1 /(m+1)}}{(m+1) t^{m /(m+1)}\left(R^{2 /(m+1)}-t^{2 /(m+1)}\right)}, \quad m \in \mathbf{Z}^{+}
$$


Given $0<\tau<1$, we select

$$
R_{m}(\tau)=\tau\left[\left(m+2+m \tau^{2}\right) /\left(m+(m+2) \tau^{2}\right)\right]^{(m+1) / 2}, \quad m \in \mathbf{Z}^{+} .
$$

Note that $\tau<R_{m}(\tau)<1$. Let $\theta_{m, \tau}$ denote the function $\theta_{m}$ with $R=R_{m}(\tau)$ and $\theta_{m, \tau}(z)|d z|$ the metric $\sigma_{m}(z)|d z|$ with the same choice of $R$. Our selection of $R_{m}(\tau)$ insures that $\theta_{m, \tau}$ is decreasing on $(0, \tau]$ and increasing on $\left[\tau, R_{m}(\tau)\right)$. The minimum value of $\theta_{m, \tau}$ on $\left(0, R_{m}(\tau)\right)$ is

$$
\boldsymbol{\theta}_{m, \tau}(\tau)=\left((m+2)+m \tau^{2}\right)^{1 / 2}\left(m+(m+2) \tau^{2}\right)^{1 / 2} / 2(m+1) \tau, \quad m \in \mathbf{Z}^{+} .
$$

Hence,

$$
T_{c}^{*}\left(\sigma_{m, \tau}(z)|d z|\right)=\theta_{m, \tau}(\delta(z, c)) \lambda_{\mathbf{B}}(z)|d z|
$$

is a metric of constant curvature -1 defined on $B\left(c, R_{m}(\tau)\right) \backslash\{c\} \subset \mathbf{B}$.

TheOREM 1. Let $X$ be a hyperbolic Riemann surface and $f \in \mathscr{F}_{m}(\mathbf{B}, X)$. If $t(f) \leqslant \tau$ $<1$, then for $z \in \mathbf{B}$,

$$
f^{X}(z)<1 / \theta_{m, \tau}(t(z, f))^{m+1} .
$$

Proof. We shall write $t(z)$ in place of $t(z, f)$. The proof will be carried out in a series of steps. Define

$$
\rho(z)|d z|=\theta_{m, \tau}(t(z)) \lambda_{\mathbf{B}}(z)|d z| .
$$

Let $B_{f}=\{z \in \mathbf{B}: t(z)=0\}$. Note that $\rho(z)|d z|$ becomes infinite at each point of $B_{f}$ and that $B_{f}$ is a discrete subset of $\mathbf{B}$. The first step is to show that $\rho(z)|d z|$ is a continuous ultrahyperbolic metric on $\mathbf{B} \backslash B_{f}$ under the assumption that $t(f)<\tau<1$. Next, it readily follows that $\rho(z)|d z|$ is a continuous $S K$ metric on $\mathbf{B} \backslash B_{f}$ in the general case $t(f) \leqslant \tau<1$. The third step is the elementary observation that $f^{*}\left(\lambda_{X}(z)|d z|\right)$ is a continuous $S K$ metric on $\mathbf{B}$ that vanishes at each point of $B_{f}$. The fourth step is to show that the product $f^{*}\left(\lambda_{X}(z)|d z|\right)^{1 /(m+2)}(\rho(z)|d z|)^{(m+1) /(m+2)}$ is a continuous $S K$ metric on all of $\mathbf{B}$; the exponents are selected so that the zeros of the first factor and the poles of the second factor cancel at points of $B_{f}$ to produce a finite-valued metric on B. Then Ahlfors' lemma [1], as refined by Heins [5], when applied to this $S K$ metric yields (1) but without strict inequality. The final step is to establish strict inequality.

(i) We begin by showing that $\rho(z)|d z|$ is a continuous ultrahyperbolic metric on $\mathbf{B} \backslash B_{f}$ under the assumption that $t(f)<\tau<1$. Obviously, $\rho(z)|d z|$ is continuous since $t(z)$ is continuous on $\mathbf{B}$. We must exhibit a supporting metric at each point of $\mathbf{B} \backslash B_{f}$. Fix $a \in \mathbf{B} \backslash B_{f}$; then $0<t(a)<\tau$. The definition of $t(a)$ implies that there is a point $c \in \mathbf{B}$ with $\delta(a, c)=t(a)$ and $t(c)=0$. We shall show that

$$
\theta_{m, \tau}(\delta(z, c)) \lambda_{\mathbf{B}}(z)|d z|=T_{c}^{*}\left(\sigma_{m, \tau}(z)|d z|\right)
$$

is a supporting metric for $\rho(z)|d z|$ at $a$. Clearly, this metric does have constant curvature -1 . Our selection of $c$ insures that $t(z) \leqslant \delta(z, c)<\tau$ for $z$ near $a$ with equality for $z=a$. Because the function $\theta_{m, \tau}$ is decreasing on the interval $(0, \tau]$, we conclude that

$$
T_{c}^{*}\left(\sigma_{m, \tau}(z)|d z|\right) \leqslant \rho(z)|d z|
$$


in a neighborhood of $a$ with equality at $a$. Hence $\rho(z)|d z|$ has a supporting metric at $a$, so it is ultrahyperbolic on $\mathbf{B} \backslash B_{f}$.

(ii) The second step is to demonstrate that $\rho(z)|d z|$ is a continuous $S K$ metric on B $\backslash B_{f}$ in case $t(f) \leqslant \tau<1$. Set $\tau_{n}=\tau+1 / n$. Define

$$
\rho_{n}(z)|d z|=\theta_{m, \tau_{n}}(t(z)) \lambda_{\mathbf{B}}(z)|d z| \text {. }
$$

Then part (i) of the proof implies that $\rho_{n}(z)|d z|$ is an ultrahyperbolic metric on B $\backslash B_{f}$ provided that $\tau_{n}<1$. This will be true for all $n$ sufficiently large. Now, $\rho_{n}(z)|d z|$ converges to $\rho(z)|d z|$ as $n \rightarrow \infty$, locally uniformly on $\mathbf{B} \backslash B_{f}$. It follows that $\rho(z)|d z|$ is a continuous $S K$ metric on $\mathbf{B} \backslash B_{f}[9]$.

(iii) Clearly, $f^{*}\left(\lambda_{X}(z)|d z|\right)$ is a continuous metric on $\mathbf{B}$ that vanishes at each point of $B_{f}$. We shall show that it has constant curvature -1 on $\mathbf{B} \backslash B_{f}$. Fix $a \in \mathbf{B} \backslash B_{f}$. Then $f$ is univalent in a neighborhood of $a$. Because curvature is a conformal invariant, it follows that $f^{*}\left(\lambda_{X}(z)|d z|\right)$ has constant curvature -1 in a neighborhood of $a$. Trivially, $f^{*}\left(\lambda_{X}(z)|d z|\right)$ can be regarded as its own supporting metric at each point of $\mathbf{B} \backslash B_{f}$, so it is an ultrahyperbolic metric on $\mathbf{B}$. Consequently, $f^{*}\left(\lambda_{X}(z)|d z|\right)$ is an $S K$ metric on $\mathbf{B}$.

(iv) From $[5$, p. 14] we conclude that

$$
\sigma(z)|d z|=f^{*}\left(\lambda_{X}(z)|d z|\right)^{1 /(m+2)}(\rho(z)|d z|)^{(m+1) /(m+2)}
$$

is a continuous $S K$ metric on $\mathbf{B} \backslash B_{f}$. Now we demonstrate that each point of $B_{f}$ is a removable singularity for the metric $\sigma(z)|d z|$ in the sense that this metric has a continuous extension to $\mathbf{B}$ and the extended metric is an $S K$ metric on $\mathbf{B}$. Let $a \in B_{f}$. Then for $z$ near $a$ we have $t(z)=\delta(z, a)$. Thus,

$$
\begin{aligned}
(\rho(z)|d z|)^{(m+1) /(m+2)} & =\left(T_{a}^{*}\left(\sigma_{m, \tau}(z)|d z|\right)\right)^{(m+1) /(m+2)} \\
& =\delta(z, a)^{-m /(m+2)} R(z)(|d z|)^{(m+1) /(m+2)},
\end{aligned}
$$

where $R(z)$ is continuous at $a$ and $R(a) \neq 0$. Also, $f=f(a)$ has a root of order $n+1$ at $a$, where $n \geqslant m$. We now assume that $X \subset \mathbf{C}$. If $X$ is a Riemann surface, then we perform similar calculations in terms of any local coordinate at the point $f(a)$. For $z$ near $a$, we have

$$
f(z)=f(a)+A(z-a)^{n+1}+B(z-a)^{n+2}+\cdots,
$$

where $A \neq 0$. Then

$$
\left|f^{\prime}(z)\right|=|z-a|^{n} g(z)=\delta(z, a)^{n}|1-\bar{a} z|^{n} g(z),
$$

where $g(z)$ is continuous and $g(a) \neq 0$. Consequently,

$$
\begin{aligned}
f^{*}\left(\lambda_{X}(z)|d z|\right)^{1 /(m+2)} & =\left(\lambda_{X}(f(z))\left|f^{\prime}(z)\right||d z|\right)^{1 /(m+2)} \\
& =\delta(z, a)^{n /(m+2)} F(z)(|d z|)^{1 /(m+2)}
\end{aligned}
$$

where $F(z)$ is continuous and $F(a) \neq 0$. Then for $z$ near $a$,

$$
\sigma(z)|d z|=\delta(z, a)^{(n-m) /(m+2)} F(z) R(z)|d z| .
$$

Because $n \geqslant m$ we see that $\sigma(z)|d z|$ has a continuous extension to $a$ and vanishes at $a$ if and only if $n>m$. Let $\sigma(z)|d z|$ denote the extension to B. Then $\sigma(z)|d z|$ is 
continuous on $\mathbf{B}$ and an $S K$ metric on $\mathbf{B} \backslash B_{f}$. Since $B_{f}$ is a discrete subset of $\mathbf{B}$, we may now conclude that $\sigma(z)|d z|$ is an $S K$ metric on all of $\mathbf{B}[5, \mathrm{pp} .9-10]$.

(v) From [5] it follows that

$$
\sigma(z)|d z| \leqslant \lambda_{\mathbf{B}}(z)|d z|
$$

and if equality holds at a single point, then $\sigma(z)|d z|=\lambda_{\mathbf{B}}(z)|d z|$. From (2) we get

$$
f^{*}\left(\lambda_{X}(z)|d z|\right)\left(\theta_{m, \tau}(t(z)) \lambda_{\mathbf{B}}(z)|d z|\right)^{m+1} \leqslant\left(\lambda_{\mathbf{B}}(z)|d z|\right)^{m+2},
$$

or

$$
f^{X}(z) \leqslant 1 / \theta_{m, \tau}(t(z))^{m+1}
$$

for $z \in \mathbf{B}$. All that remains is to demonstrate that strict inequality holds in (3). If equality holds in (3) at some point of $\mathbf{B}$, then equality holds in (2) at the same point. This implies that $\sigma(z)|d z|=\lambda_{\mathbf{B}}(z)|d z|$. Let us show that this is impossible. Consider $a \in B_{f}$; such a point exists since $t(f) \leqslant \tau<1$. From part (iv) of the proof we conclude that

$$
\rho(z)|d z|=T_{a}^{*}\left(\sigma_{m, \tau}(z)|d z|\right)
$$

for $z$ near $a, z \neq a$. Thus,

$$
f^{*}\left(\lambda_{X}(z)|d z|\right)^{1 /(m+2)}\left(T_{a}^{*}\left(\sigma_{m, \tau}(z)|d z|\right)\right)^{(m+1) /(m+2)}=\lambda_{\mathbf{B}}(z)|d z|
$$

in a deleted neighborhood of $a$, so that the product has constant curvature -1 in a deleted neighborhood of $a$. Then Lemma 1 implies that

$$
f^{*}\left(\lambda_{X}(z)|d z|\right)=T_{a}^{*}\left(\sigma_{m, \tau}(z)|d z|\right)
$$

in a deleted neighborhood of $a$. This is impossible since the left-hand side of (4) vanishes at $a$ while the right-hand side becomes infinite at $a$. Thus, strict inequality must hold in (3).

REMARK. For $f \in \mathscr{F}_{1}(\mathbf{B}, X)$, where $X$ is a hyperbolic Riemann surface, the principle of hyperbolic metric implies that

$$
f^{*}\left(\lambda_{X}(z)|d z|\right) \leqslant \lambda_{\mathbf{B}}(z)|d z| \text { or } f^{X}(z) \leqslant 1,
$$

with equality if and only if $f$ is an analytic universal covering of $\mathbf{B}$ onto $X$ [8]. Because $\theta_{m, \tau}(\tau)>1$, Theorem 1 can be viewed as a refinement of the principle of hyperbolic metric. In particular, for $X=\mathbf{B}$, Theorem 1 refines the invariant form of Schwarz' lemma. Different refinements of the principle of hyperbolic metric occur in [8 and 9].

Now we can obtain a lower bound for $\mathcal{T}_{m, X}(\alpha)$. Define

$$
h_{m}(\tau)=1 / \theta_{m, \tau}(\tau), \quad m \in \mathbf{Z}^{+} .
$$

Then $h_{m}$ is strictly increasing on $[0,1]$, fixes 0 and 1 and

$$
h_{m}^{-1}(\alpha)=\alpha\left[\frac{m(m+2)}{2(m+1)^{2}-\left(m^{2}+2 m+2\right) \alpha^{2}+2(m+1)\left[\alpha^{4}-\left(m^{2}+2 m+2\right) \alpha^{2}+(m+1)^{2}\right]^{1 / 2}}\right]^{1 / 2}
$$

THEOREM 2. If $\alpha \in(0,1]$ and $X$ is any hyperbolic Riemann surface, then $\mathcal{T}_{m, X}(\alpha) \geqslant$ $h_{m}^{-1}\left(\alpha^{1 /(m+1)}\right)$. 
Proof. If this result were false, then there would exist $f \in \mathscr{F}_{m}(\mathbf{B}, X)$ such that $f^{X}(0) \geqslant \alpha$ and $\tau=t(f)<h_{m}^{-1}\left(\alpha^{1 /(m+1)}\right) \leqslant 1$. Now if we apply Theorem 1 to the function $f$ at the point $z=0$, then we obtain

$$
\alpha \leqslant f^{X}(0)<1 / \theta_{m, \tau}(t(0, f))^{m+1} \leqslant 1 / \theta_{m, \tau}(\tau)^{m+1}=h_{m}(\tau)^{m+1}<\alpha,
$$

a contradiction.

REMARK. For $\alpha=1$ and any $m \in \mathbf{Z}^{+}$this theorem is sharp. It gives $\mathscr{T}_{m, X}(1) \geqslant$ $h_{m}^{-1}(1)=1$. But trivially, $\mathcal{T}_{m, X}(1) \leqslant 1$, so $\mathcal{T}_{m, X}(1)=1$. But this result is trivial. If $f \in \mathscr{F}_{m}(\mathbf{B}, X)$ and $f^{X}(0)=1$, then $f$ must be a universal covering. Hence, $f$ is locally schlicht and $t(f)=1$. Also, for a fixed $\alpha \in(0,1], \lim _{m \rightarrow \infty} h_{m}^{-1}\left(\alpha^{1 /(m+1)}\right)=1$. This agrees with the fact that $\sigma_{\infty, X}(\alpha)=1$.

A lower bound for $\mathcal{T}_{1, \mathbf{B}}(\alpha)$ is implicit in the work of Landau. He proved that if $f$ is a holomorphic self-mapping of $\mathbf{B}$ and $\left|f^{\prime}(0)\right| \geqslant \alpha$, then $f$ is univalent in $B(0, \rho)$, where $\rho=\alpha /\left(1+\left(1-\alpha^{2}\right)^{1 / 2}\right)$. This result is contained in [6, p. 37] and is sharp. If $B(z)=z(\alpha-z) /(1-\alpha z)$, then $B^{\prime}(0)=\alpha$ and $B^{\prime}(\rho)=0$. This implies that $t(0, B)$ $=\rho$. Also, this result implies that if $f: \mathbf{B} \rightarrow \mathbf{B}$ is analytic and $f^{\mathbf{B}}(0) \geqslant \alpha$, then $t(0, f) \geqslant \rho$. Therefore,

$$
\mathcal{T}_{1, \mathbf{B}}(\alpha) \geqslant \alpha /\left(1+\sqrt{1-\alpha^{2}}\right) .
$$

However, direct calculation shows that

$$
h_{1}^{-1}(\sqrt{\alpha})>\alpha /\left(1+\sqrt{1-\alpha^{2}}\right), \quad \alpha \in(0,1) .
$$

Thus, the lower bound given by Theorem 2 improves the lower bound that is implicit in Landau's work.

In our definition of $\mathcal{T}_{m, X}(\alpha)$ we require that $X$ be a hyperbolic Riemann surface. If

$$
\mathcal{T}_{m, \mathbf{C}}(\alpha)=\inf \left\{t(f): f \in \mathscr{F}_{m}(\mathbf{B}, \mathbf{C}) \text { and }\left|f^{\prime}(0)\right| \geqslant \alpha\right\},
$$

then it is elementary to see that $\mathcal{T}_{1, \mathbf{C}}(\alpha)=0$. In fact, let $Z$ be a discrete subset of $\mathbf{B}$ such that if $z \in Z$, then there exists $w \in Z, w \neq z$, with $\delta(z, w) \leqslant \varepsilon$, where $\varepsilon>0$ is a given number. We assume that $0 \notin Z$. Then there exists a holomorphic function $f$ on B which vanishes at each point of $Z$ and at no other point of $\mathbf{B}$. We may even assume that $f^{\prime}(0) \neq 0$. Let $g=f^{2}$. Then $t(g) \leqslant \varepsilon$. By multiplying $g$ by a suitable scalar, we can make its derivative at the origin be any value $\alpha \in(0, \infty)$. Hence, $\mathcal{T}_{1, \mathbf{C}}(\alpha) \leqslant \varepsilon$, so $\mathcal{T}_{1, \mathbf{C}}(\alpha)=0$. Thus, for $X=\mathbf{C}$ the domain Bloch constants can be trivial.

5. Upper bound for $\mathcal{T}_{m, \mathrm{~B}}(\alpha)$. Ahlfors and Grunsky [3] gave an upper bound for the classical Bloch constant that is conjectured to be sharp. They obtained this upper bound by constructing a specific function. The basic idea involved in this construction was generalized in [9] and [10] to obtain upper bounds for various euclidean, hyperbolic and spherical Bloch constants. This same generalization can be used to obtain upper bounds for $\mathcal{T}_{m, \mathbf{B}}(\alpha)$. We just outline the idea here; for details the reader should consult [9].

For $q \in\left[0, \frac{1}{3}\right)$ let $\Delta_{q}$ be the regular circular triangle which has all interior angles of size $\pi q$ and vertices at the points $1, \omega$ and $\omega^{2}$, where $\omega=\exp (2 \pi i / 3)$. Set

$$
R_{q}=\left[\sin \pi\left(\frac{5}{6}+\frac{q}{2}\right) / \sin \pi\left(\frac{1}{6}+\frac{q}{2}\right)\right]^{1 / 2}, \quad q \in\left[0, \frac{1}{3}\right) .
$$


The value of $R_{q}$ is selected so that $R_{q} \Delta_{q}=\left\{R_{q} z: z \in \Delta_{q}\right\}$ is a hyperbolic triangle in B. Let $f_{q}: \mathbf{B} \rightarrow \Delta_{q}$ be the unique conformal mapping that satisfies the conditions $f\left(\omega^{j}\right)=\omega^{j}(j=0,1,2)$. Then $f_{q}(0)=0$ and

$$
f_{q}^{\prime}(0)=\Gamma\left(\frac{5}{6}+\frac{q}{2}\right) \Gamma\left(\frac{2}{3}\right) / \Gamma\left(\frac{1}{6}+\frac{q}{2}\right) \Gamma\left(\frac{4}{3}\right) .
$$

Define

$$
g_{m, q}(z)=R_{q} f_{q} \circ f_{q /(m+1)}^{-1}\left(z / R_{q /(m+1)}\right)
$$

for $q \in\left[0, \frac{1}{3}\right)$ and $m \in \mathbf{Z}^{+}$. Then $g_{m, q}$ is a conformal mapping of $R_{q /(m+1)} \Delta_{q /(m+1)}$ onto $R_{q} \Delta_{q}$ which sends 0 to 0 and makes the vertices correspond. Also,

$$
\alpha_{m, q}=g_{m, q}^{\prime}(0)=\frac{R_{q} \Gamma(5 / 6+q / 2) \Gamma(1 / 6+q / 2(m+1))}{R_{q /(m+1)} \Gamma(1 / 6+q / 2) \Gamma(5 / 6+q / 2(m+1))} .
$$

Now we can construct the functions which produce upper bounds for $\mathcal{T}_{m, \mathbf{B}}(\alpha)$ for certain values of $\alpha$. For $m \in \mathbf{Z}^{+}$we consider $g_{m, 1 / n}$, where $n \geqslant 4$. The triangle $R_{1 / n} \Delta_{1 / n}, n \geqslant 4$, leads to a triangulation of $\mathbf{B}$ that is obtained by starting with $R_{1 / n} \Delta_{1 / n}$ and applying successive reflections in its sides and in the sides of newly formed triangles. The Schwarz reflection principle in conjunction with this observation about triangulations of $\mathbf{B}$ implies that $g_{m, 1 / n}$ extends to a holomorphic function defined on all of $\mathbf{B}$; we use the same notation for the extended function. Then $g_{m, 1 / n}$ is locally schlicht in $\mathbf{B}$ except at each vertex of the triangulation of $\mathbf{B}$ that is induced from $R_{1 / n(m+1)} \Delta_{1 / n(m+1)}$. At each such vertex the function $g_{m, 1 / n}$ assumes a value with exact multiplicity $m+1$; the value assumed at such a vertex is one of the vertices of the triangulation of $\mathbf{B}$ induced from $R_{1 / n} \Delta_{1 / n}$. Thus, $g_{m, 1 / n} \in \mathscr{F}_{m}(\mathbf{B}, \mathbf{B})$ and clearly $t\left(g_{m, 1 / n}\right) \leqslant R_{1 / n(m+1)}$. This gives an upper bound for $\mathscr{T}_{m, \mathrm{~B}}(\alpha)$ for the countable number of values $\alpha=\alpha_{m, 1 / n}, n \geqslant 4$. It would be interesting to give examples for $\alpha \neq \alpha_{m, 1 / n}$. For $m=1$ we get $\mathscr{T}_{m, \mathbf{B}}\left(\alpha_{1,1 / n}\right) \leqslant R_{1 / 2 n}$. The following table gives upper and lower bounds for $\mathcal{T}_{m, \mathbf{B}}\left(\alpha_{1,1 / n}\right)$ for selected values of $n$.

$\begin{array}{rccc}n & \alpha_{1,1 / n} & \begin{array}{c}\text { Lower Bound } \\ h_{1}^{-1}\left(\sqrt{\alpha_{1,1 / n}}\right)\end{array} & \begin{array}{c}\text { Upper Bound } \\ R_{1 / 2 n}\end{array} \\ 4 & .7171 & .5101 & .6982 \\ 5 & .8413 & .6172 & .7546 \\ 6 & .8964 & .6817 & .7928 \\ 7 & .9265 & .7263 & .8206 \\ 8 & .9449 & .7594 & .8417 \\ 9 & .9571 & .7850 & .8584 \\ 10 & .9656 & .8056 & .8718 \\ 25 & .9947 & .9192 & .9470 \\ 50 & .9987 & .9589 & .9732 \\ 100 & .9997 & .9793 & .9865\end{array}$

6. A related open problem. Let $X$ be a Riemann surface and $f: \mathbf{B} \rightarrow X$ an analytic function. For $z \in \mathbf{B}$ let $s(z, f)$ be the maximum value of $r$ such that $f$ is schlicht in $B(z, r)$; set $s(z, f)=0$ in case $f$ is not univalent in any neighborhood of $z$. Clearly, 
$s(z, f) \leqslant t(z, f)$. Let $s(f)=\sup \{s(z, f): z \in \mathbf{B}\}$. For $\alpha \in(0,1]$ and a hyperbolic Riemann surface $X$ let

$$
\delta_{X}(\alpha)=\inf \left\{s(f): f \in \mathscr{F}_{1}(\mathbf{B}, X) \text { and } f^{X}(0) \geqslant \alpha\right\} .
$$

Of course, one could also introduce constants $\S_{m, X}(\alpha)$ by restricting consideration to functions $f \in \mathscr{F}_{m}(\mathbf{B}, X)$. It would be interesting to determine bounds for $\mathscr{S}_{X}(\alpha)$.

The constant $\delta_{X}(1)$ has already been considered, but in a different guise. If $f \in \mathscr{F}_{1}(\mathbf{B}, X)$ and $f^{X}(0)=1$, then $f$ must be an analytic universal covering of $\mathbf{B}$ onto $X$. Let $\Gamma$ be the associated group of cover transformations; $\Gamma$ is a fixed-point free Fuchsian group. Then the work of Harvey [4] and Marden [7] implies that there is a constant $r>0$, independent of the hyperbolic Riemann surface $X$, such that any normal polygon for $\Gamma$ contains a hyperbolic ball of radius $r$. Sturm and Shinnar [11] gave an explicit lower bound for $r$. Actually, this result is true for an arbitrary Fuchsian group; that is, the group $\Gamma$ is allowed to contain elliptic elements. Now $f$ is injective on the interior of any normal polygon, so it follows that $s(f) \geqslant \tanh (r / 2)$. Recently, Yamada [14] has shown that the largest possible value for $r$ is $\sinh ^{-1}(2 / \sqrt{3})$. Consequently,

$$
\delta_{X}(1) \geqslant \tanh \left(\frac{1}{2} \sinh ^{-1}\left(\frac{2}{\sqrt{3}}\right)\right)=\frac{2+\sqrt{7}-\sqrt{3}}{2+\sqrt{7}+\sqrt{3}}=.45685 \ldots,
$$

with equality for the thrice punctured sphere. The explicit determination of related constants is given in [13].

Sharp lower bounds for $\delta_{X}(\alpha), \alpha \in(0,1)$, are not known. Is it possible to obtain a lower bound for this constant by using the differential-geometric method of Ahlfors and Heins? Yamada used a different method to derive the sharp lower bound for $\delta_{X}(1)$.

ADDED IN PROOF. Recently, the author has obtained lower bounds for $\widehat{S}_{X}(\alpha)$ for various classes of Riemann surfaces (Marden constants for Bloch and normal functions, J. Analyse Math., to appear). The lower bound is independent of $\alpha \in(0,1]$ and of $X$, provided $X$ supports nonconstant Bloch functions. Related results are obtained for surfaces which support nonconstant normal functions.

\section{REFERENCES}

1. L. V. Ahlfors, An extension of Schwarz's lemma, Trans. Amer. Math. Soc. 43 (1938), 359-364.

2. __ Conformal invariants, Topics in Geometric Function Theory, McGraw-Hill, New York, 1973.

3. L. V. Ahlfors and H. Grunsky, Über die Blochsche Konstante, Math. Z. 42 (1937), 671-673.

4. W. Harvey, Chabauty spaces of discrete groups, Discontinuous Groups and Riemann Surfaces (L. Greenberg, editor), Ann. of Math. Studies, no. 79, Princeton Univ. Press, Princeton, N. J., 1974, pp. 239-246.

5. M. Heins, On a class of conformal metrics, Nagoya Math. J. 21 (1962), 1-60.

6. Selected topics in the classical theory of functions of a complex variable, Holt, Rinehart, and Winston, New York, 1962.

7. A. Marden, Universal properties of Fuchsian groups in the Poincare metric, Discontinuous Groups and Riemann Surfaces (L. Greenberg, editor), Ann. of Math. Studies, no. 79, Princeton Univ. Press, Princeton, N. J., 1974, pp. 315-339.

8. C. D. Minda, The hyperbolic metric and coverings of Riemann surfaces, Pacific J. Math. 84 (1979), $171-182$. 
9. , Bloch constants, J. Analyse Math. (to appear).

10. __ Bloch constants for meromorphic functions, Math. Z. 181 (1982), 83-92.

11. J. Sturm and M. Shinnar, The maximal inscribed ball of a Fuchsian group, Discontinuous Groups and Riemann Surfaces (L. Greenberg, editor), Ann. of Math. Studies, no. 79, Princeton Univ. Press, Princeton, N. J., 1974, pp. 439-443.

12. M. Tsuji, Potential theory in modern function theory, Maruzen, Tokyo, 1959.

13. A. Yamada, On Marden's universal constant of Fuchsian groups, Kodai Math. J. 4 (1981), 266-277.

14. , On Marden's universal constant of Fuchsian groups. II (preprint).

Department of Mathematical Sciences, University of Cincinnati, Cincinnati, Ohio 45221 